\title{
Design of Wireless Automatic Synchronization for the Low-Frequency Coded Ground Penetrating Radar
}

\author{
Zhenghuan Xia, ${ }^{1,2}$ Qunying Zhang, ${ }^{1}$ Shengbo Ye, ${ }^{1}$ Zhiwu Xu, \\ Jie Chen, ${ }^{1}$ Guangyou Fang, ${ }^{1}$ and Hejun Yin ${ }^{3}$ \\ ${ }^{1}$ Key Laboratory of Electromagnetic Radiation and Sensing Technology, Chinese Academy of Sciences, Beijing 100190, China \\ ${ }^{2}$ University of Chinese Academy of Sciences, Beijing 100049, China \\ ${ }^{3}$ Chinese Academy of Sciences, Beijing 100864, China \\ Correspondence should be addressed to Zhenghuan Xia; maxwell_xia@126.com
}

Received 5 February 2015; Revised 26 May 2015; Accepted 27 May 2015

Academic Editor: Ahmed El Wakil

Copyright (C) 2015 Zhenghuan Xia et al. This is an open access article distributed under the Creative Commons Attribution License, which permits unrestricted use, distribution, and reproduction in any medium, provided the original work is properly cited.

Low-frequency coded ground penetrating radar (GPR) with a pair of wire dipole antennas has some advantages for deep detection. Due to the large distance between the two antennas, the synchronization design is a major challenge of implementing the GPR system. This paper proposes a simple and stable wireless automatic synchronization method based on our developed GPR system, which does not need any synchronization chips or modules and reduces the cost of the hardware system. The transmitter omits the synchronization preamble and pseudorandom binary sequence (PRBS) at an appropriate time interval, while receiver automatically estimates the synchronization time and receives the returned signal from the underground targets. All the processes are performed in a single FPGA. The performance of the proposed synchronization method is validated with experiment.

\section{Introduction}

Low-frequency ground penetrating radar (GPR) is an advanced geophysical technique that is rapidly and widely applied in deep detection $[1,2]$. In order to achieve high signal-to-noise ratio (SNR), pseudorandom coded signals had been applied in some detection applications, such as through-the-wall tracking and life detection [3-6]. Meanwhile, pseudorandom coded signals had also been used in GPR to obtain the deeper detection $[7,8]$. In practical applications, wire dipole antennas are more suitable for lowfrequency GPR system with large transmit power. However, the synchronization between transmitter and receiver is a major challenge.

Synchronization with long cables introduces severe interference to the returned signals, which could mask the returned signals from the underground targets. Synchronization with fibers increases the power consumption, complicates the structure, and increases the cost of the radar system. Additionally, it is terribly difficult to carry out experiments with complicate and heavy low-frequency GPR in polar glacier and high tableland $[9,10]$.

In order to simplify the low-frequency GPR structure and reduce the cost of hardware system, a simple and efficient wireless automatic synchronization method is proposed. Firstly, our developed low-frequency coded GPR will be described briefly. Secondly, the wireless automatic synchronization method will be discussed in detail, and the estimation error of synchronization time will be analyzed. Thirdly, the performance of the proposed method of wireless automatic synchronization will be validated with experiment.

\section{Description of Our Developed Low-Frequency Coded GPR}

Our developed low-frequency coded GPR consists of a pair of resistively loaded wire dipole antennas with a length of $12 \mathrm{~m}$, a transmitter with a peak power of $200 \mathrm{~W}$, and a receiver with two sampling channels, which is shown in Figure 1. In 


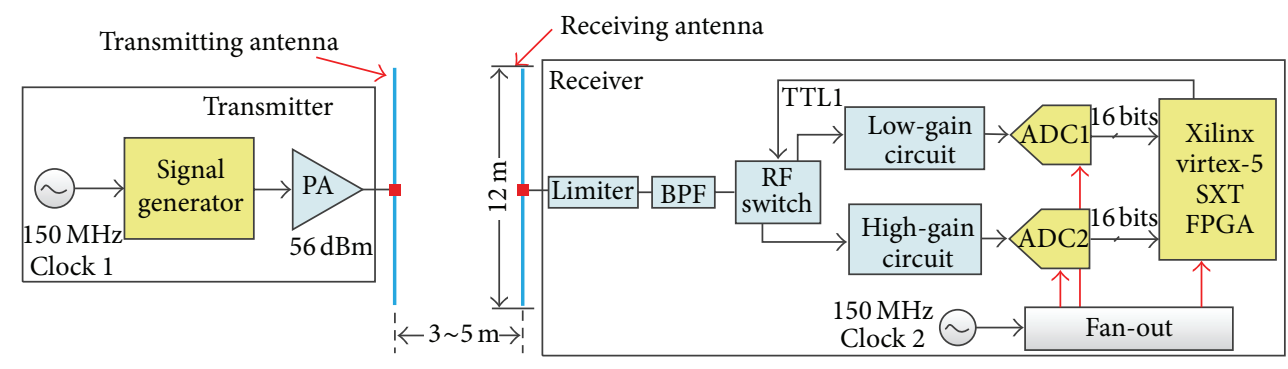

FIGURE 1: The architecture of our developed low-frequency coded GPR.

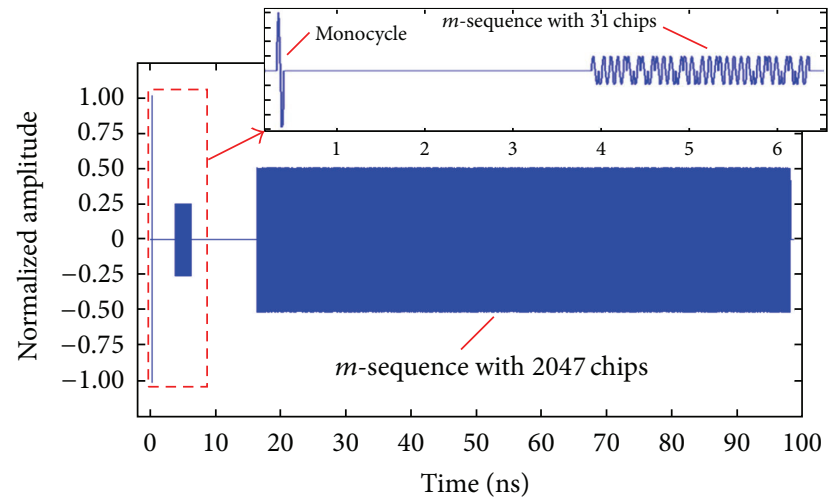

FIGURE 2: The transmit signal from the signal generator.

practical applications, the distance between the two antennas is from $3 \mathrm{~m}$ to $5 \mathrm{~m}$.

2.1. Transmitter. One steady-state $150 \mathrm{MHz}$ clock source is the reference clock of the signal generator. The coded signals produced by the signal generator are amplified by a power amplifier (PA) with a maximal peak power of $56 \mathrm{dBm}$ to feed the transmitting antenna. In order to achieve good synchronization without affecting the radar echo, the transmit signals consist of a synchronization preamble and $m$-sequence of 2047 chips at a time interval of 10 us as shown in Figure 2. The synchronization preamble includes a monocycle and $m$-sequence of 31 chips at a time interval of 3.5 us. The monocycle is used to estimate coarsely the time of arrival of the direct wave of the $m$-sequence with 31 chips, which is used to determine accurately the synchronization time. The $m$ sequence of 2047 chips is used to obtain the impulse response of the deep targets with low side-lobes.

Additionally, the peak power of the monocycle, $m$ sequence with 31 chips, and $m$-sequence of 2047 chips are $56 \mathrm{dBm}, 44 \mathrm{dBm}$, and $50 \mathrm{dBm}$, respectively. Both the two $m$ sequences are modulated with a $12 \mathrm{MHz}$ sine wave, and the width of each chip of $m$-sequence is about $80 \mathrm{~ns}$.

2.2. Receiver. Another steady-state $150 \mathrm{MHz}$ clock source is used as the main clock of the receiver. As shown in Figure 1, the $150 \mathrm{MHz}$ clock is sent to the fan-out chip to generate three synchronized clocks, which are used as the clocks of the two
TABLE 1: Parameters of the low-frequency coded GPR.

\begin{tabular}{lc}
\hline Parameter & Value \\
\hline Width of the monocycle & $80 \mathrm{~ns}$ \\
Width of each chip & $80 \mathrm{~ns}$ \\
Length of the long $m$-sequence & $2047 \mathrm{chips}$ \\
Peak power & $50 \mathrm{dBm}$ \\
Receiver sensitivity & $-115 \mathrm{dBm}$ \\
Real-time sampling rate of ADC & $150 \mathrm{Mbps}$ \\
The linear average factor & 512 \\
The scanning rate & $8 \mathrm{scans} / \mathrm{s}$ \\
Pulse repeated period & $200 \mathrm{us}$ \\
Maximal detection time window & $4 \mathrm{us}$ \\
\hline
\end{tabular}

ADCs and FPGA. The limiter is used to prevent the receiver from damage. During the estimation of synchronization time, the synchronization preamble is switched to the lowgain circuit with $3 \mathrm{~dB}$ gain and sampled by the first $\mathrm{ADC}$ at the sampling rate of $150 \mathrm{MHz}$, and FPGA estimates the synchronization time. Once the synchronization time has been estimated, the radar echo is switched to high-gain circuit with $30 \mathrm{~dB}$ and is sampled by the second ADC at the same sampling rate of $150 \mathrm{MHz}$. Then the FPGA performs the cross-correlation between the sampled radar echo and the responding reference signal to obtain the impulse response of the detection scenarios.

Additionally, the main parameters of the proposed lowfrequency coded GPR are summarized in Table 1.

\section{Design of Wireless Automatic Synchronization}

The transmitter omits the coded signals at a pulse repeated period (PRP) of 200 us, and the receiver estimates accurately the synchronization time and receives the radar echoes. The responding reference signals of the two $m$-sequences can be obtained in close-loop situation as shown in Figure 3, and the two reference signals are stored in two on-chip RAMs of the FPGA. The reference signal of the $m$-sequence with 31 chips is denoted as $m(i)$, and the reference signal of the $m$ sequence with 2047 chips is denoted as $m_{l}(i)$. The architecture 


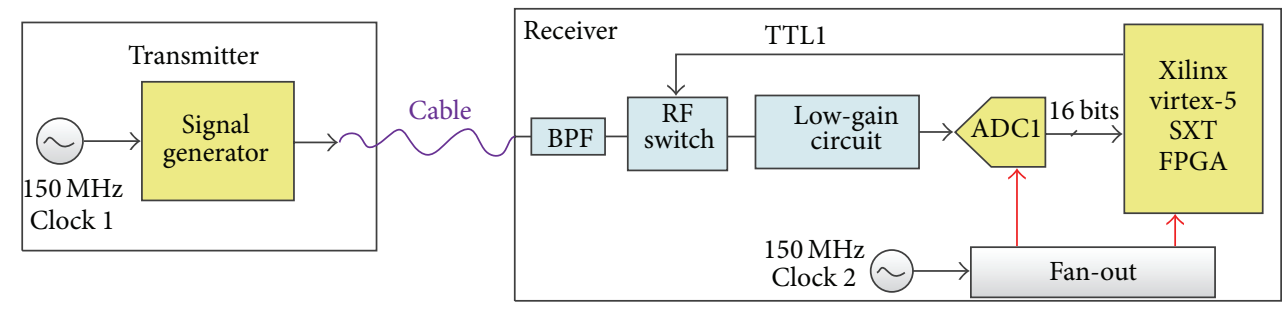

FIGURE 3: Obtain the reference signal of the $m$-sequence in close-loop situation.

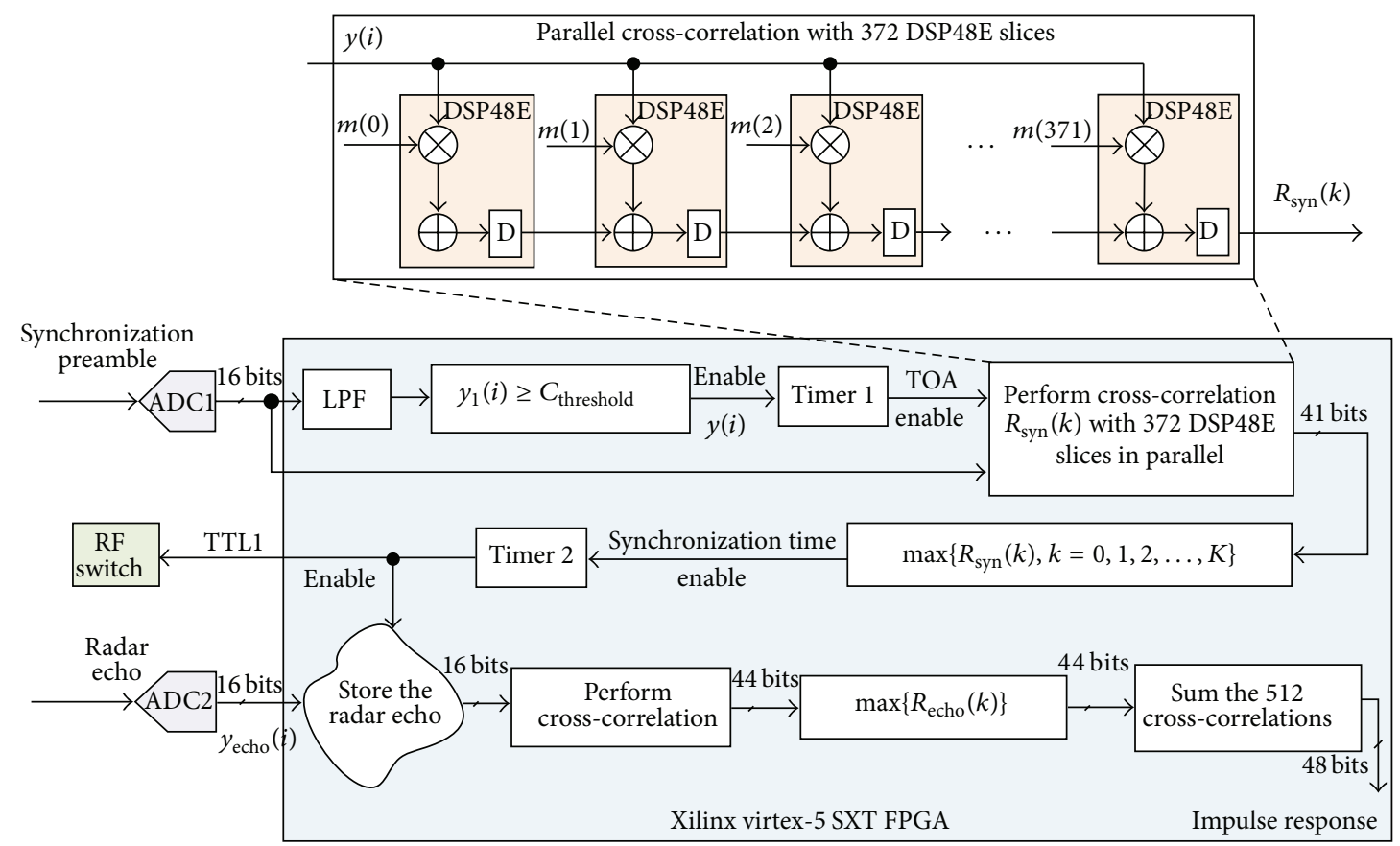

FIGURE 4: Estimation of the synchronization time based on FPGA.

of wireless automatic synchronization in the receiver is shown in Figure 4.

3.1. Estimation of the Synchronization Time. Firstly, the direct wave of the monocycle sampled by the first ADC, which is denoted as $y(i)$, is filtered by the digital low pass filter (LPF) to remove the noise and compared with the threshold $C_{\text {threshold }}$. The threshold $C_{\text {threshold }}$ should be chosen according to the amplitude of the direct wave and the level of the noise or clutter. For our low-frequency GPR system, the maximum clutter or noise is about $42 \mathrm{mV}$, and the amplitude of the direct wave is about $610 \mathrm{mV}$ when the distance between the two antennas is $3 \mathrm{~m}$. When the distance is increased to $5 \mathrm{~m}$, the amplitude of the direct wave is decreased to about $220 \mathrm{mV}$. Thus the threshold is fixed at $150 \mathrm{mV}$ in this work, and the distance between the two antennas should be less than $5 \mathrm{~m}$ in practical applications.

Secondly, when the filtered signal $y_{l}(i)$ is more than the threshold, the first timer Timer 1 is enabled to count. The TOA of the $m$-sequence with 31 chips comes when the timer Timer 1 counts to 3.5 us. At this time, the sampled signal $y(i)$ could be considered coarsely as the direct wave of the $m$-sequence of 31 chips and sent to the parallel crosscorrelation module to obtain the cross-correlation $R_{\text {syn }}(k)$ as

$$
R_{\text {syn }}(k)=\sum_{i=0}^{371} m(i) \cdot y(k+i), \quad k=0,1, \ldots, K,
$$

where $m(i)$ is the reference signal of the $m$-sequence of 31 chips and stored in a RAM of FPGA. $M$-sequence with 31 chips lasts for about 2.48 us and the sampling clock of ADC is $150 \mathrm{MHz}$, and the length of the reference signal $m(i)$ is 372. The clock of the parallel cross-correlation module is $150 \mathrm{MHz}$ synchronized with the sampling clock of ADCs. The pipeline delay and throughput rate of the parallel crosscorrelation module are 372 clock periods and $150 \mathrm{Msps}$, respectively. Then FPGA can find the maximum value of the cross-correlation by comparison between the two adjacent cross-correlations in a time window of 2.48 us. Ultimately, the synchronization time can be determined by the maximal value of cross-correlation.

Thirdly, when the synchronization time is estimated, the timer Timer 2 is enabled to count and the radar echo is switched to the high-gain circuit and sampled by the second 


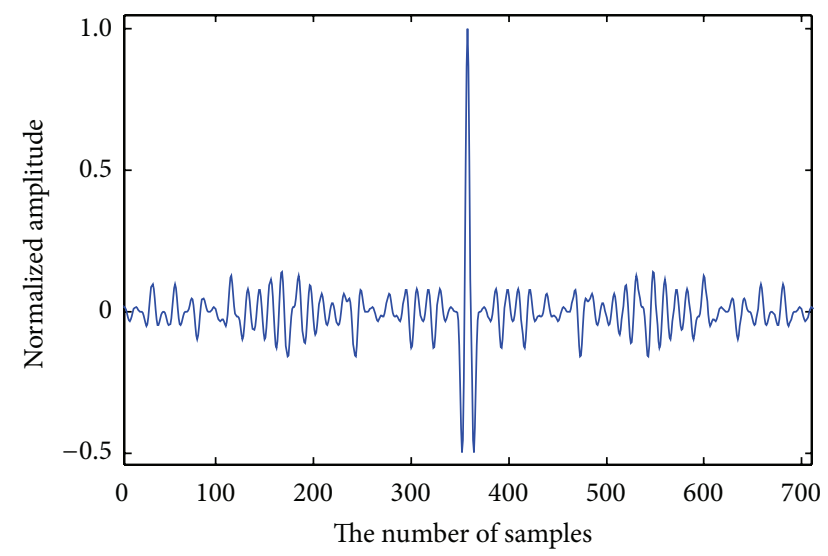

(a)

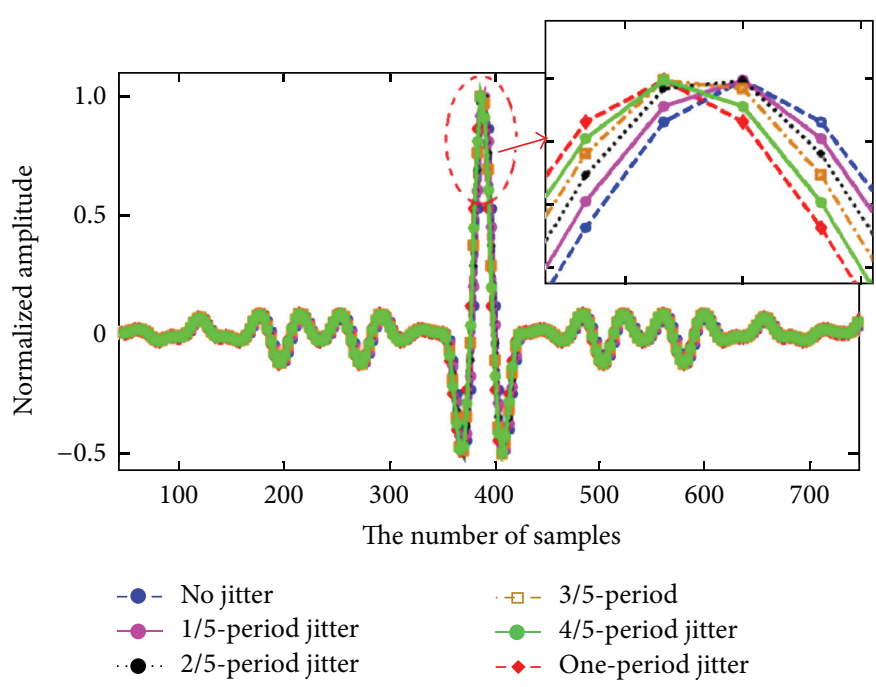

(b)

Figure 5: Cross-correlation of the $m$-sequence with 31 chips in close-loop situation. (a) Cross-correlation of the sampled $m$-sequence with 31 chips. (b) Cross-correlations of the sampled $m$-sequence with unstable initial sampling time.

ADC when the timer Timer 2 counts to 10 us. Then the crosscorrelation $R_{\text {echo }}(k)$ of the echo is performed to obtain the impulse response of detection scenarios. In order to perform correctly the linear averaging to improve the SNR of the radar echoes, the maximal value of the cross-correlation $R_{\text {echo }}(k)$ in each returned signal should be found and then performs addition among the adjacent 512 traces to obtain the ultimate impulse response of the underground targets.

3.2. Analysis of the Estimation Error. As shown in Figure 1, the two reference clocks, Clock 1 and Clock 2, are not synchronized, which induces the estimation error of synchronization time. It is equivalent to the fact that the estimation error comes from the random jitter of the initial sampling time of the receiver, and the maximal jitter is one period of Clock 2. In close-loop situation as shown in Figure 3, all the reference signals of the cross-correlation have been sampled at a sampling clock of Clock 2 and stored in FPGA beforehand. According to (1), the cross-correlation of the sampled $m$-sequence with 31 chips in close-loop situation is shown in Figure 5(a). Figure 5(b) shows the estimation errors of synchronization time come from different jitter of the initial sampling time of the receiver, which indicates that the maximal estimation error is also one period of Clock 2 when the jitter is more than a half period of Clock 2. Then the estimation error will affect the initial acquisition time for the radar echo.

Figure 6 shows the comparison between the crosscorrelation of the received $m$-sequence of 2047 chips with one-period estimation error of synchronization time and that of no estimation error. It can be seen that the main-lobe to side-lobe ratio of the two cross-correlations is almost the same because of the high sampling rate relative to the lowfrequency $m$-sequence. However, the location of the maximal value of the cross-correlation with one-period error is ahead one period of that of the cross-correlation without error.

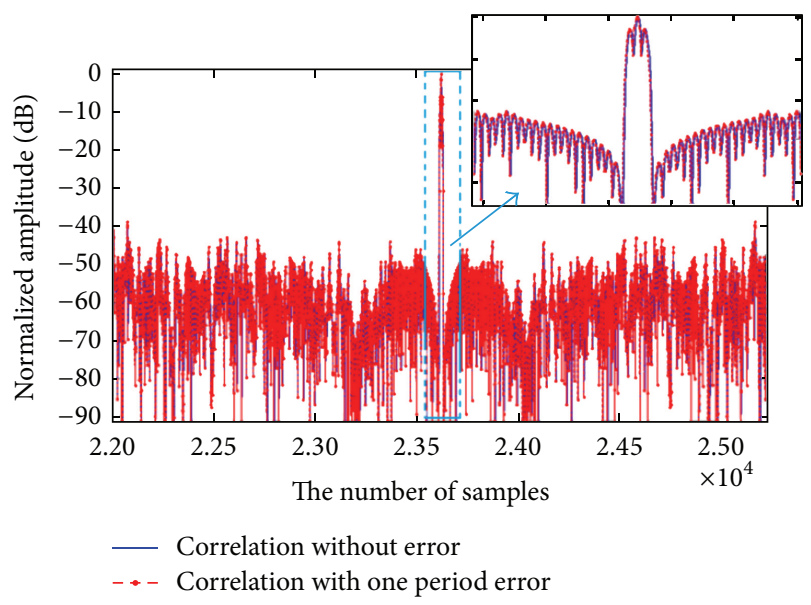

FIgUre 6: Comparison between the cross-correlation of the $m$ sequence with 2047 chips with one-period estimation error and that of $m$-sequence with no estimation error.

In order to perform correctly the linear averaging with 512 traces, the maximal value of the cross-correlation should be found firstly, and the 512 additions are performed from the maximal value of the 512 cross-correlations.

\section{Experiments and Results}

The typical configuration for the low-frequency GPR is shown in Figure 7. The distance between the two wire antennas is about $3.8 \mathrm{~m}$, and the synchronization is implemented with two methods: high-performance ORTEL's fiber models (including OTS-1RefR-100 and OTS-1RefT-100 [11]) and the proposed wireless automatic synchronization method. For this wireless automatic synchronization, the received direct wave of the monocycle as the blue line is shown in 


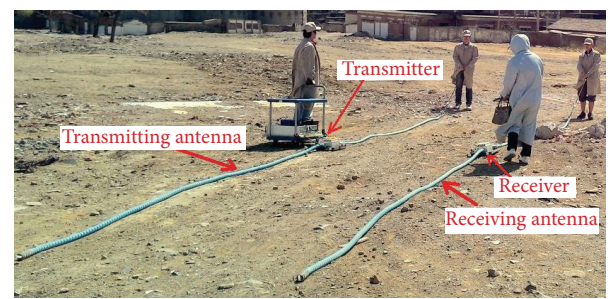

FIgURE 7: The typical configuration for the low-frequency coded GPR.

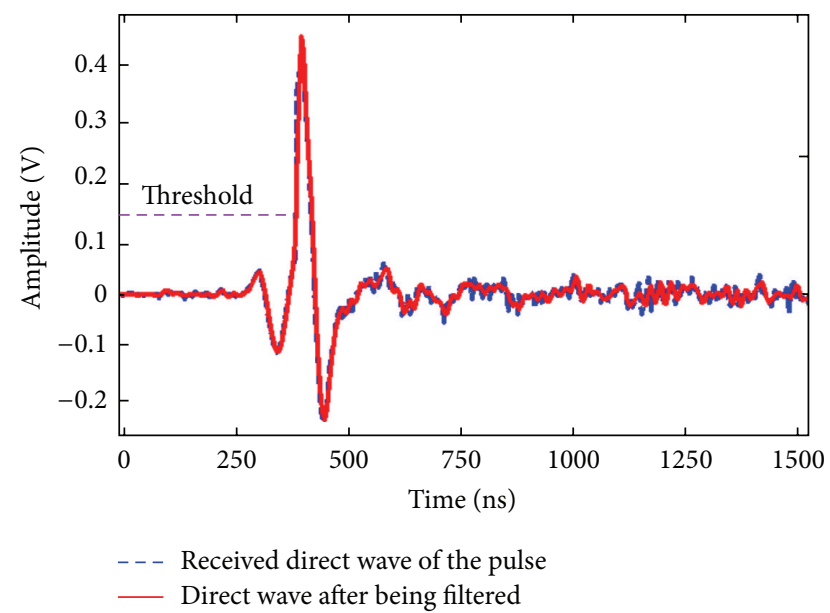

(a)

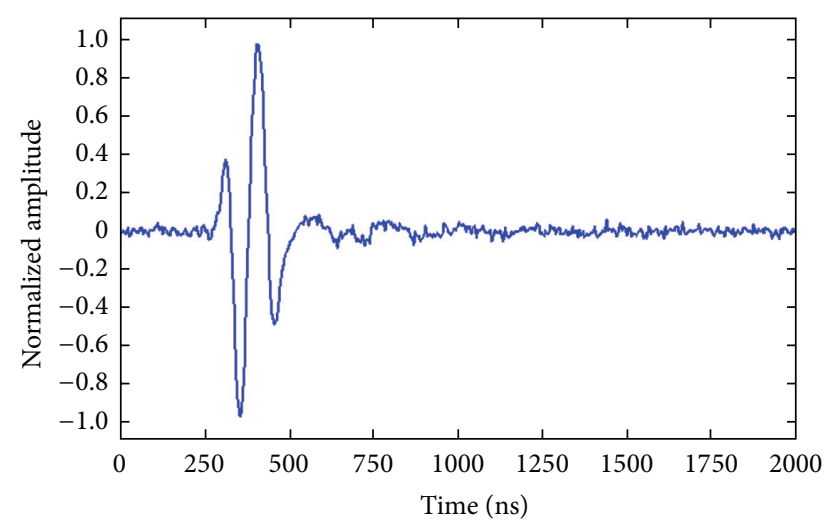

(b)

FIGURE 8: Received synchronization preamble. (a) The direct wave of the monocycle. (b) The cross-correlation of the direct wave of the $m$-sequence with 31 chips.

Figure 8(a), while the filtered signal as the red line is shown in Figure 8(a). The direct wave of the $m$-sequence with 31 chips is received, and its cross-correlation can be obtained as shown in Figure 8(b). The synchronization time can be estimated by the maximal value of the cross-correlation.

The red line as shown in Figure 9(a) is the obtained impulse response with the proposed wireless automatic synchronization method, while the blue line in Figure 9(a) is the obtained impulse response with the fiber modules.

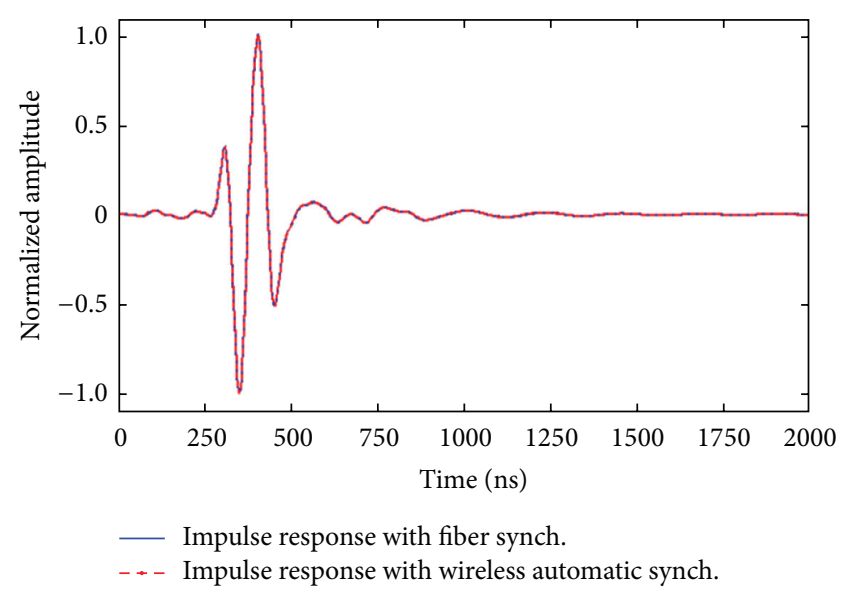

(a)

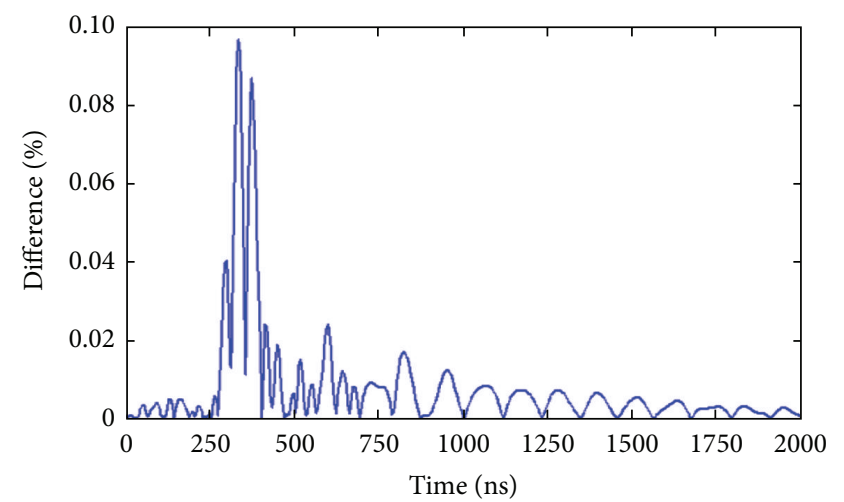

(b)

FIGURE 9: The obtained impulse responses with the two methods. (a) Two impulse responses. (b) The difference between them.

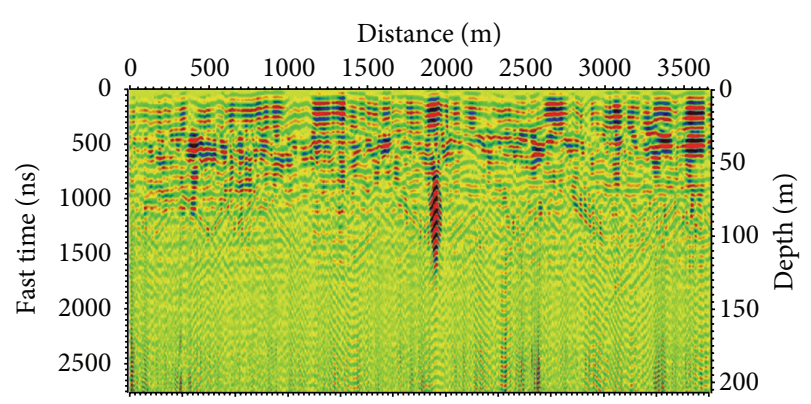

FIGURE 10: The detection result with the low-frequency coded GPR, and the dielectric constant is 8 .

The difference between the two impulse responses is shown in Figure 9(b), which indicates that the two impulse responses are almost same, while different clutter and noise have been received in different time. Figure 10 shows the responding detection result with the proposed low-frequency coded GPR in desert. It can be seen that some clear layers in depth of $45 \mathrm{~m}$ and $70 \mathrm{~m}$ can be detected. 


\section{Conclusion}

This paper presents a wireless automatic synchronization method for the low-frequency coded GPR without any other synchronization chips or modules, which simplify the architecture of the GPR system and reduce the hardware cost. Although the maximum synchronization error with the proposed method is one sampling period of ADC-clock, it degrades hardly the main-lobe to side-lobe ratio of pulse compression due to the high sampling rate relative to the low-frequency $m$-sequence. In order to reduce the effect from the synchronization error farther, the ADC-clock can be increased to an appropriate value to satisfy the requirement of different applications, such as $400 \mathrm{MHz}$ or $1 \mathrm{GHz}$.

\section{Conflict of Interests}

The authors declare that there is no conflict of interests regarding the publication of this paper.

\section{Acknowledgment}

This work was supported by the National High Technology Research and Development Program of China (863 Program: 2012AA121901).

\section{References}

[1] V. Utsi, "Design of a GPR for deep investigations," in Proceedings of the 4th International Workshop on Advanced Ground Penetrating Radar, pp. 222-225, Aula Magna Partenope, June 2007.

[2] L. Fu, S. Liu, L. Liu, and L. Lei, "Development of an airborne ground penetrating radar system: antenna design, laboratory experiment, and numerical simulation," IEEE Journal of Selected Topics in Applied Earth Observations and Remote Sensing, vol. 7, no. 3, pp. 761-766, 2014.

[3] Z. Xia, G. Fang, S. Ye, Q. Zhang, C. Chen, and H. Yin, "A novel handheld pseudo random coded UWB radar for human sensing applications," IEICE Electronics Express, vol. 11, no. 23, pp. 1-7, 2014.

[4] S. R. J. Axelsson, "Noise radar using random phase and frequency modulation," IEEE Transactions on Geoscience and Remote Sensing, vol. 42, no. 11, pp. 2370-2384, 2004.

[5] A. Nezirović, A. G. Yarovoy, and L. P. Ligthart, "Signal processing for improved detection of trapped victims using UWB radar," IEEE Transactions on Geoscience and Remote Sensing, vol. 48, no. 4, pp. 2005-2014, 2010.

[6] Z. Xia, G. Fang, S. Ye et al., "Design of modulated m-sequence ultrawideband radar for life detection," in Proceedings of the 15th International Conference on Ground Penetrating Radar (GPR '14), pp. 960-963, IEEE, Brussels, Belgium, July 2014.

[7] F. Nicollin, Y. Barbin, W. Kofman et al., "An HF bi-phase shift keying radar: Application to ice sounding in Western Alps and Spitsbergen glaciers," IEEE Transactions on Geoscience and Remote Sensing, vol. 30, no. 5, pp. 1025-1033, 1992.

[8] G. Fang and M. Pipan, "Design of a low frequency ultrawideband (UWB) antenna and its applications in ground penetrating radar (GPR) system," in Proceedings of the tenth International Conference on Ground penetrating Radar, pp. 109111, Delft, The Netherlands, 2004.
[9] M. Carnevale and J. Hager, "Low frequency GPR in difficult terrain," in Proceedings of the 4th International Workshop on Advanced Ground Penetrating Radar, pp. 68-73, Aula Magna Partenope, Italy, June 2007.

[10] M. Tallini, D. Ranalli, M. Scozzafava, and G. Manacorda, "Testing a new low-frequency GPR antenna on karst environments of central Italy," in Proceedings of the Tenth International Conference Ground Penetrating Radar (GPR '04), pp. 133-135, June 2004.

[11] http://www.emcore.com/. 

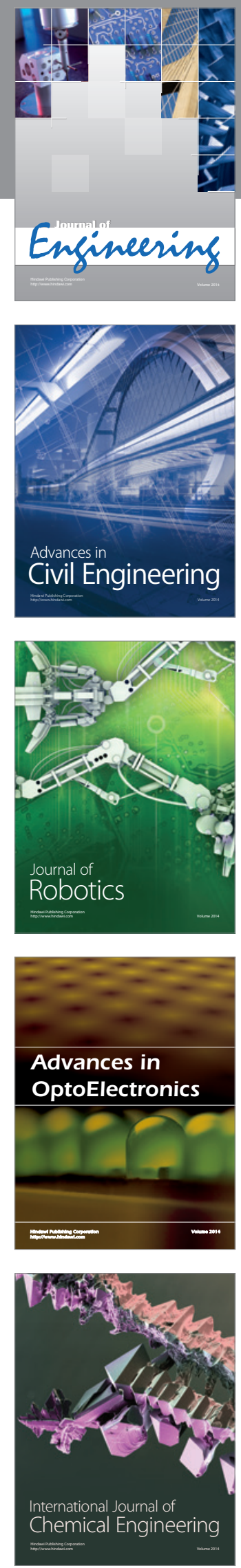

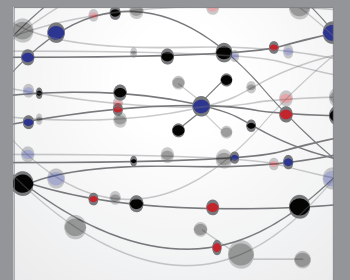

The Scientific World Journal
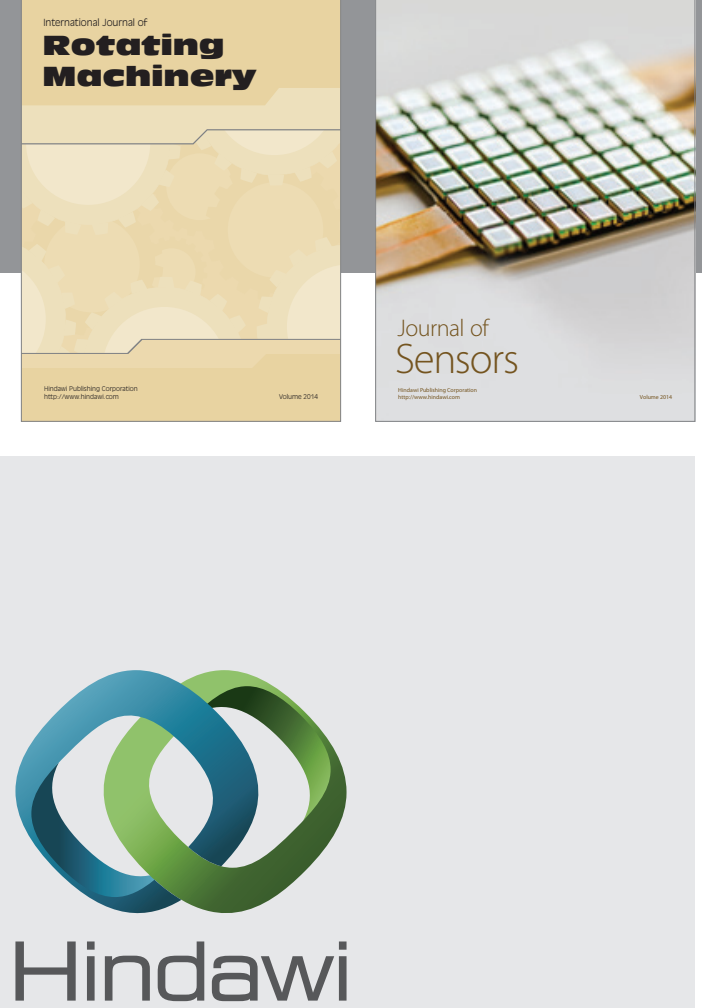

Submit your manuscripts at http://www.hindawi.com
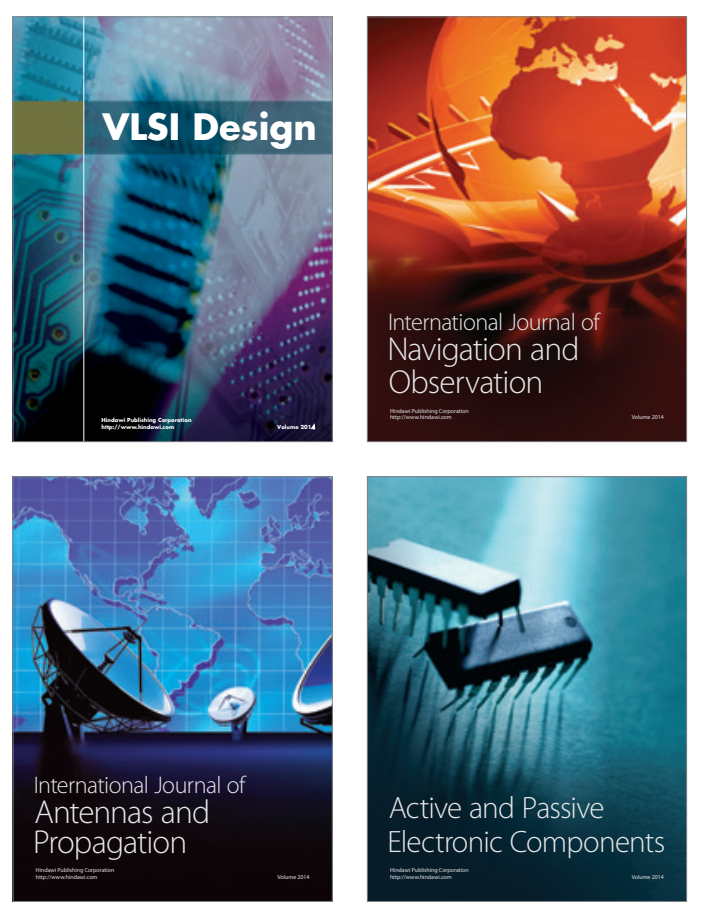
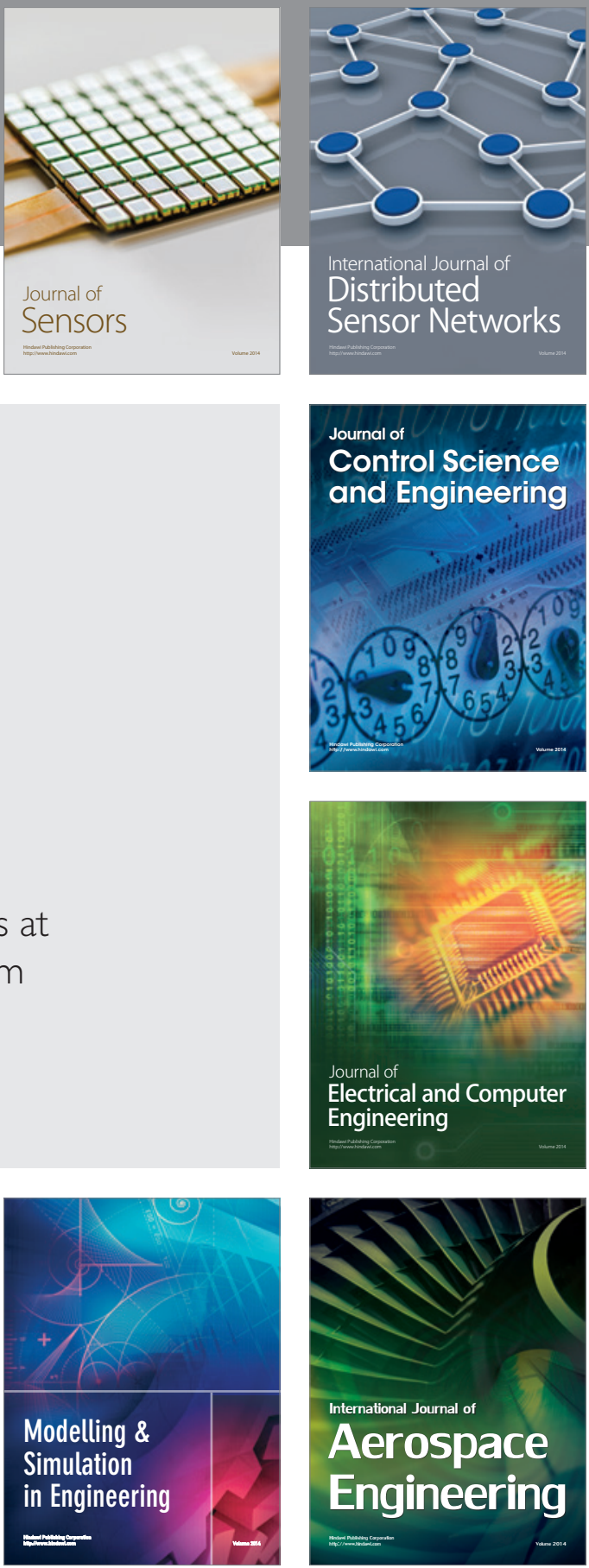

Journal of

Control Science

and Engineering
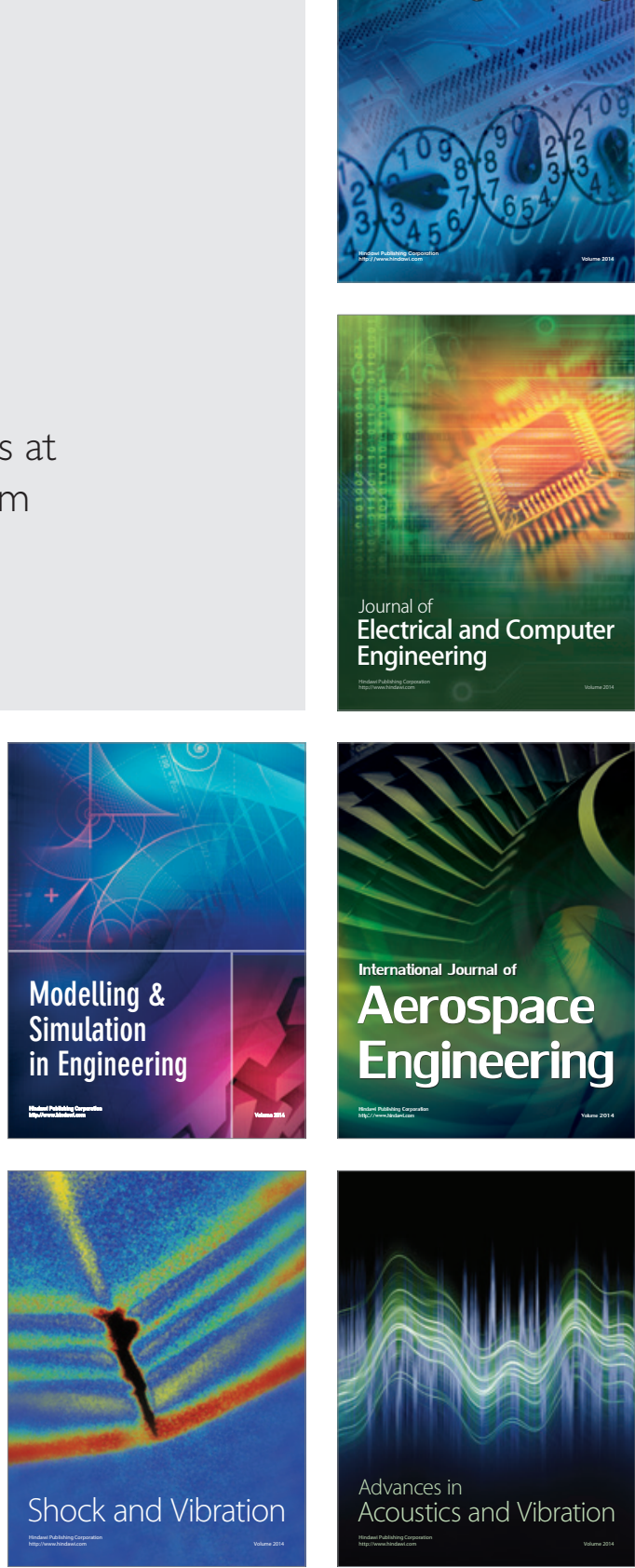